\title{
Global identification and analysis of long non- coding RNAs in diploid strawberry Fragaria vesca during flower and fruit development
}

\author{
Chunying Kang ${ }^{2^{*}}$ and Zhongchi Liu ${ }^{1 *}$
}

\begin{abstract}
Background: Long non-coding RNAs (IncRNAs) are a new class of regulatory molecules with roles in diverse biological processes. While much effort has been invested in the analysis of IncRNAs from established plant models Arabidopsis, maize, and rice, almost nothing is known about IncRNAs from fruit crops, including those in the Rosaceae family.

Results: Here, we present a genome-scale identification and characterization of IncRNAs from a diploid strawberry, Fragaria vesca, based on rich RNA-seq datasets from 35 different flower and fruit tissues. 5,884 Fve-IncRNAs derived from 3,862 loci were identified. These IncRNAs were carefully cataloged based on expression level and whether or not they contain repetitive sequences or generate small RNAs. About one fourth of them are termed highconfidence IncRNAs (hc-IncRNAs) because they are expressed at a level of FPKM higher than 2 and produce neither small RNAs nor contain repetitive sequence. To identify regulatory interactions between IncRNAs and their potential protein-coding (PC) gene targets, pairs of IncRNAs and PC genes with positively or negatively correlated expression trends were identified based on their expression; these pairs may be candidates of cis-or trans-acting IncRNAs and their targets. Finally, blast searches within plant species indicate that IncRNAs are not well conserved.
\end{abstract}

Conclusions: Our study identifies a large number of tissue-specifically expressed IncRNAs in $F$. vesca, thereby highlighting their potential contributions to strawberry flower and fruit development and paving the way for future functional studies.

Keywords: LncRNA, Strawberry, Flower, Fruit, RNA-seq, Fragaria vesca, Rosaceae

\section{Background}

With the advent of new genomic techniques such as tiling arrays and next generation sequencing $[1,2]$, non-coding RNAs are increasingly identified and recognized as an integral and functional component of the genome. While some non-coding RNAs perform housekeeping functions, such as tRNAs, rRNAs and small nuclear RNAs, others such as microRNAs and small interfering RNAs (siRNA) play critical regulatory roles during development or stress responses [3, 4]. Non-coding RNAs with a length greater than 200bp are defined as long non-coding RNAs (lncRNAs). The lncRNAs can be grouped into three

\footnotetext{
* Correspondence: ckang@mail.hzau.edu.cn; zliu@umd.edu

${ }^{2}$ College of Horticulture and Forestry Sciences, Huazhong Agricultural University, Wuhan 430070, China

'Department of Cell Biology and Molecular Genetics, University of Maryland, College Park, MD 20742, USA
}

subclasses: 1) long non-coding natural antisense transcripts (lnc-NATs), 2) intronic lncRNAs, and 3) intergenic IncRNAs. Like protein-coding (PC) genes, a majority of lncRNAs are transcribed by RNA polymerase II with a $5^{\prime}$ cap and a 3' poly-A tail in animals [5]. In plants, however, lncRNAs can be transcribed by PolII, IV, and V, therefore some may lack poly-A tails [6-9].

A growing number of reports revealed lncRNAs from animals, especially human; they are involved in diverse biological processes, such as development, cellular differentiation, and diseases including cancers. LncRNAs may serve as diagnostic markers or even therapeutic targets [10-15]. Studies of animal IncRNAs showed that lncRNAs function through a number of mechanisms. First, lncRNAs act in epigenetic regulation of gene expression. For instance, the mammalian XIST RNA initiates $\mathrm{X}$ chromosome inactivation in cis to equalize gene 
expression between males and females [16, 17]. HOTAIR (HOX antisense intergenic RNA) is able to mediate transcriptional repression of $H O X$ loci in trans by modulating histone methylation [18]. Second, lncRNAs may directly interact with proteins to titrate their functions and are thus called 'decoys'. A well-known example is the IncRNA named TERRA, which was demonstrated to be the natural ligand and inhibitor of telomerase [19]. Third, lncRNAs may act as a scaffold to form a complex with other proteins. Together, lncRNAs were demonstrated to play bona fide and essential roles in animals.

In contrast, there are only a handful of reports on the functions of lncRNAs in plants. FLOWERING LOCUS C (FLC) is a key flowering repressor in the vernalization pathway. To ensure epigenetic silencing of $F L C$, a lnc-NAT named COOLAIR (COLD INDUCED LONG ANTISENSE INTRAGENIC RNA) and an intronic lncRNA called COLDAIR (COLD ASSISTED INTRONIC NONCODING RNA) could be induced after vernalization treatment to gradually repress the expression of $F L C$ by promoting methylation $[7,20]$. Another intergenic lncRNA induced by phosphate starvation was found in Medicago truncatula (Mt4), Arabidopsis thaliana (IPS1, INDUCED BY PHOSPHATE STARVATION1 and At4), tomato (Lycopersicon esculentum L.; TPSI1, TOMATO PHOSPHATE STARVATIONINDUCED GENE 1), and rice (Oryza sativa; OsPI1, ORYZA SATIVA PHOSPHATE-LIMITATION INDUCIBLE GENE 1) [21-24]. Further analysis indicated that IPS1 acts as a decoy of miRNA-399 and allows the accumulation of its target gene transcripts [25]. A third intergenic lncRNA called LDMAR (LONG-DAY-SPECIFIC MALE-FERTILITYASSOCIATED RNA) is required for normal pollen development in rice under long day conditions [26]. A number of lncRNAs are differentially expressed under stress stimuli in Arabidopsis [9]. Despite limited reports on the mechanisms of plant lncRNA function, it is evident that plant lncRNAs play vital roles in developmental and stress responses. This realization combined with the advent of next-generation sequencing has prompted tremendous efforts and investments in identifying lncRNAs in a wide range of organisms $[5,27-35]$.

F. vesca, the woodland strawberry, is becoming a new model organism for both octoploid cultivated strawberry (Fragaria $x$ ananassa) and other members of the Rosaceae, a family that includes many fruit trees. F. vesca has a short life cycle, small stature, facile transformation, and small and sequenced genome $(2 n=14,240 \mathrm{Mb})$ [36-38]. Further, transcriptomic data of various tissues in the cultivated strawberry were available [39]. Of significant interest is the strawberry fruit, which is developed from the receptacle (the stem tip that supports the flower) [40]. Toward the identification of molecular mechanisms of strawberry floral and fruit development, we first generated comprehensive RNA-seq datasets from 35 distinct $F$. vesca floral and fruit tissues at different developmental stages to profile genome-wide expression of PC genes [41, 42]. In current work, we seek to identify lncRNAs from strawberry floral and fruit transcriptomes with the goal of uncovering lncRNAs that function in flower and fruit development. In total, 5,884 lncRNAs derived from 3,862 loci were identified from the $F$. vesca flower and fruit trancriptome dataset. Further analysis indicated that these lncRNAs are similar to PC genes in terms of gene structure and transcriptional regulation. However, lncRNAs also show a number of distinctions from PC genes. For example, a large number of lncRNAs are precursors to small RNAs, and their sequences are much less conserved than PC genes. In an effort to identify potential regulatory target genes of lncRNAs, we used expression correlation between lncRNAs and the PC genes in the $F$. vesca genome, taking advantage of the RNA-seq data from a large number of different floral and fruit tissues. Both positive and negatively correlated lncRNA-PC gene pairs are identified. Our analysis provided the first look at the lncRNA landscape in a fruit crop and laid the foundation for future studies of lncRNA function in strawberry. To facilitate the study of lncRNAs and data sharing, our F. vesca IncRNAs can be accessed from the Strawberry Genome Resources (SGR) website [43] as a new track in gBrowse (http://bioinformatics.towson.edu/strawberry/) as well as from Genome Database for Rosaceae (GDR; https://www.rosaceae.org) [44].

\section{Results}

Identification of $F$. vesca IncRNAs from flower and fruit RNA-seq datasets

To globally identify lncRNAs in the F.vesca genome, we utilized RNA-seq datasets generated from 35 distinct floral and fruit tissues plus two vegetative tissues (seedlings and young leaves), which were isolated from a $7^{\text {th }}$ generation inbred line of $F$. vesca (Yellow Wonder 5AF7 or YW5AF7) (Additional file 2) [41, 42]. There were two biological replicates for each tissue type, and hence 74 RNA-seq libraries in total, amounting to about 2.1 billion total single-end reads of $51 \mathrm{bp}$. The analysis pipeline is shown in Fig. 1. In brief, each library was aligned individually by Tophat 2 in order to preserve junction reads. Known and novel transcripts were assembled by cufflinks based on uniquely mapped reads and, finally, all assemblies were combined by cuffmerge and then compared to the annotations by cuffcompare to characterize each transcript.

In total, 15,027 candidate noncoding transcripts $(8,709$ loci) in class "u" (unknown, intergenic), "o" (generic exonic overlap with a reference transcript), " $x$ " (overlap 


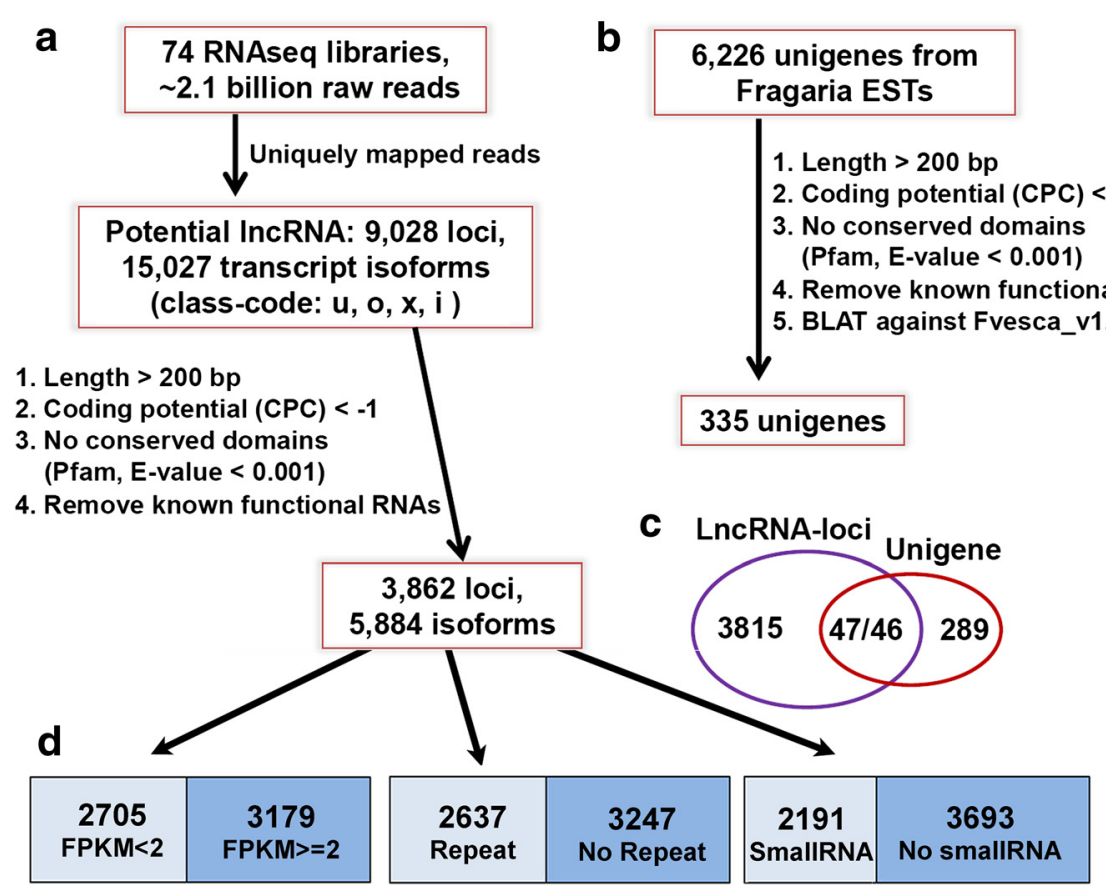

Fig. 1 Summary of the workflows used for identifying strawberry IncRNAs. a IncRNA identification from $F$. vesca RNA-seq datasets. b IncRNA identification from unigenes. $\mathbf{c}$ Venn diagram comparing IncRNAs identified in (a) and (b). d Different classifications of fve-IncRNAs based on expression levels, repeats, and precursors of small RNAs. hc-IncRNAs: high confidence IncRNAs

with a reference transcript in the opposite orientation), and "i" (intronic) were subjected to further filtering (Fig. 1a, Table 1). The transcripts are defined as lncRNAs if they are longer than 200bp, are non-coding, and are not a member of known functional RNA classes (tRNA, rRNA, snRNA, miRNA) (Fig. 1a). A relatively stringent non-coding standard was used. First, the Coding Potential Calculator was used to discriminate non-coding from coding transcripts as it takes into account several biologically meaningful sequence features with high accuracy [45]; a non-coding transcript has a coding potential score (CPC) lower than “-1". Second, to reduce false positive, any short peptide coded by "non-coding transcript" should contain no conserved protein domains (Pfam database). As a result, 5,884 transcript isoforms in 3,862 loci were identified as lncRNAs (Additional files 3, 4 and 5).

In addition to the RNA-seq data, we mined existing ESTs from Fragaria. A large number of raw EST datasets derived from both diploid Fragaria vesca and octoploid Fragaria $\times$ ananassa were found in the NCBI dbEST database, but unigenes from GDR (Genome Database for Rosaceae) were chosen for lncRNA discovery as unigenes do not contain low quality ESTs or redundant ESTs [46]. The filtering criteria were similar to the IncRNA discovery from RNA-seq data (Fig. 1b) and led to the identification of 335 lncRNAs out of 6,226 unigenes (Additional files 6 and 7). Interestingly, only 46 of the 334 lncRNAs are in common with the RNA-seqderived lncRNAs (Fig. 1c). We then compared the

Table 1 The number of IncRNAs in different classes before and after filtering

\begin{tabular}{|c|c|c|c|c|}
\hline \multirow[b]{2}{*}{ Class-code } & \multicolumn{2}{|l|}{ Isoform } & \multicolumn{2}{|l|}{ Locus } \\
\hline & Before $^{a}$ & After $^{\mathrm{b}}$ & Before $^{a}$ & After $^{b}$ \\
\hline u (unknown or intergenic region) & 7,203 & 3,763 & 4,726 & 2,575 \\
\hline o (overlapped with existed gene with a dramatic difference in gene structures) & 5,125 & 625 & 2,824 & 464 \\
\hline x (overlapped with existed gene in an opposite direction) & 2,658 & 1,471 & 1,438 & 902 \\
\hline i (located in introns) & 41 & 25 & 40 & 24 \\
\hline Total & 15,027 & 5,884 & $8,709^{c}$ & $3,862^{c}$ \\
\hline
\end{tabular}

Notes:

${ }^{a}$ The number of original isoforms and loci predicted by Cufflinks

${ }^{\mathrm{b}}$ The number of IncRNAs after filtering

"Total" number is smaller than the sum of the column, because certain loci have multiple isoforms with different class codes 
remaining 289 unigene-derived lncRNAs with the $F$. vesca genome annotation and found that a majority of them are from annotated PC loci on either the same or opposite strand sometimes covering the introns. A lack of over-lap between RNA-seq- and unigene-derived lncRNAs suggests that the identification of lncRNAs is far from saturation, partly due to a lack of strand orientation information from our RNA-seq reads.

\section{Identification of high confidence (hc)-IncRNAs}

The 5,884 lncRNAs identified from the $F$. vesca RNAseq data were further characterized based on expression levels and whether or not they contain repeats or generate small RNAs (Fig. 1d; Additional file 8). First, 3,179 lncRNAs are expressed at higher than 2 FPKM (Fragments Per Kilobase of exon per Million fragments mapped) in at least one of the tissue types in both replicates; the remaining 2,705 lncRNAs are expressed at lower than 2 FPKM. Second, lncRNAs that overlapped with transposable elements and/or repeats were identified by RepeatMasker and RepeatScout, respectively (see Methods). In total, 3,247 transcripts with a percentage of repetitive sequences lower than $10 \%$ were classified as "no repeat" (Fig. 1d; Additional file 8).

The filtering pipeline (Fig. 1a) has removed known pre-miRNA transcripts, but a small quantity of the remaining lncRNAs may still encode previously unknown miRNAs. Moreover, some lncRNAs would generate short hairpin RNAs and siRNAs involved in epigenetic regulation [30, 47]. To distinguish those small RNA-generating lncRNAs, we used the small RNA-seq dataset from nine strawberry tissue types [48]. In total, 224 million small RNA-seq reads between $18 \mathrm{bp}$ and $30 \mathrm{bp}$ were mapped against the 5,884 lncRNAs by Bowtie $1.7 .7 \%$ of the small RNA reads mapped perfectly to the lncRNAs; the majority (58 \%) of these small RNAs were $21 \mathrm{bp}$ in length. This is in sharp contrast with the total small RNA reads with 24 bp small RNA as the most abundant species (Additional file 1: Figure S1). We thus identified 2,191 potentially small RNA-generating lncRNAs, defined as having higher than 10 small RNA reads mapped to the lncRNA locus (Fig. 1d; Additional file 8).

When all three filtering criteria were applied, only 1,556 high confidence IncRNAs (hc-lncRNAs) were obtained (Additional file 8). They are expressed at a relatively high level ( $>2$ FPKM), contain no repeats, and do not produce small RNAs.

\section{Characterization of strawberry IncRNAs}

The 32,831 predicted PC genes from the F. vesca genome are evenly distributed across chromosomes (Additional file 1: Figure S2A). This is in contrast to some other genomes, such as maize and soybean, which have lower gene densities in the pericentromeric regions. Like the PC genes of $F$. vesca, 5,884 Fve-lncRNAs are also distributed evenly across the seven chromosomes (Additional file 1: Figure S2A). However, Fve-lncRNAs also exhibit marked differences from Fve-PC genes. First, lncRNAs have fewer exons (Additional file 1: Figure S2B). The majority of Fve-lncRNAs ( $65 \%$ of lncRNAs and $75 \%$ of hc-lncRNAs) possess only one or two exons, while only $38 \%$ of the PC genes have $<=2$ exons. Second, lncRNAs are generally shorter than $\mathrm{PC}$ transcripts (Additional file 1 : Figure S2C). Third, a larger number of $\mathrm{PC}$ genes are expressed at a higher level than lncRNA-coding loci, according to the FPKM extracted from the output of a single cuffdiff run (Additional file 1: Figure S2D).

\section{LncRNAs are expressed in specific tissues and stages}

During the cuffdiff run, both differentially expressed (DE) loci and their isoforms (alternatively spliced transcripts at each locus) were examined by pairwise comparisons between successive developmental stages of the same tissue types (q-value $<0.01$, fold change $>2$, Additional files 9 and 10). In these comparisons, anthers and embryos have the most DE lncRNA isoforms and loci; very few DE lncRNAs were found in receptacle cortex and pith (see details in the Additional files 9 and 10). In total, 1617 isoforms from 1619 loci showed differential expression between at least two different tissue types. The Z-score was obtained for each of these $\mathrm{DE}$ loci based on averaged FPKM of two biological replicates. A Z-score-based heatmap was made by hierarchical clustering across all tissues (Fig. 2a). Overall, a large number of lncRNAs were specific to one tissue at one specific stage. The biggest cluster of loci was exclusively expressed in the mature pollen. Relatively more IncRNAs were uniquely expressed in Anther_9, Anther_12, and Embryo_3. It may be that Anther_12 and mature pollen share some specifically expressed lncRNAs since pollen was collected from stage 12 anthers. A similar gene expression trend was also observed when looking at the expression of all isoforms (Additional file 1: Figure S3). Hence, lncRNA expression is spatially and temporally regulated. The IS (JensenShannon) specificity score was used to estimate the degree of tissue specificity. When a gene is expressed exclusively in a particular tissue, its JS score equals to 1. The distribution of the JS score shows that more lncRNAs have a higher score than PC genes $(P<2.2 \times 10$ -16 , Kolmogorov-Smirnov test), suggesting that a higher percentage of lncRNAs were more exclusively expressed (Fig. 2b).

To validate the RNA-seq data, ten lncRNAs with anther- or mature pollen-specific expression were selected for verification by RT-PCR (Additional file 11). These IncRNAs have a class_code " $\mathrm{u}$ " or " $\mathrm{x}$ " with FPKM 


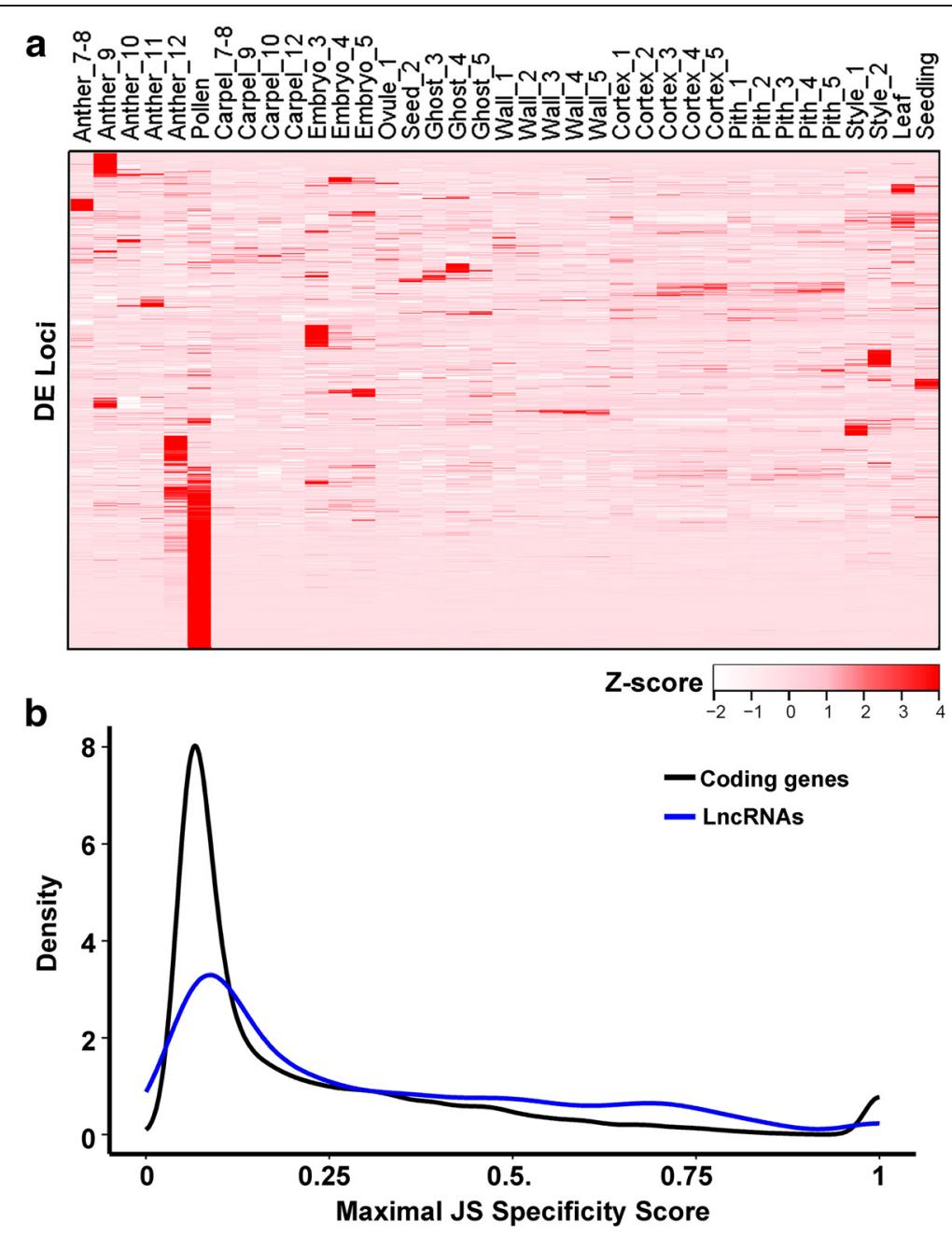

Fig. 2 Heatmaps showing tissue-specific expression of differentially expressed IncRNAs. a Heatmap showing expression patterns of 1,619 differentially expressed IncRNAs. They are defined as differentially expressed when q-value $<0.01$, fold change $>2$ in one of the pairwise comparisons. Z-score obtained from averaged FPKM of two replicates was used. $\mathbf{b}$ The distributions of the maximal JS (Jensen-Shannon) specificity score of coding genes and IncRNA-loci, respectively

starting from 4. The expected amplicons were observed for all ten lncRNAs (Fig. 3a), but some primers also amplified non-specific bands in the same tissue (XLOC_010117 and XLOC_011745) or different bands between tissues (XLOC_035638 and XLOC_003838). We further tested the expression levels of six lncRNAs by quantitative RT-PCR. XLOC_028671 is highly expressed in stage 7-9 anthers, just before or at the stage of microspore tetrad formation (Fig. 3b). XLOC_019639 and XLOC_030226 are more abundant in stage 10 anthers, the stage at which the tapetum cells start to degenerate (Fig. 3c, d). XLOC_023242 is highly expressed in stage 11 anthers (Fig. 3e). Both XLOC_036386 and XLOC_033366 are predominantly expressed in mature pollen and slightly expressed in stage 12 anthers (Fig. 3f, g). In general, the qRT-PCR results (black bars) are consistent with the RNA-seq results estimated by Cufflinks (red lines) (Fig. 3).

\section{Expression correlation of IncRNAs with neighboring PC genes}

LncRNAs regulate gene expression via a number of mechanisms including the regulation of neighboring loci in cis $[8,49]$. We thus examined the correlation in expression between lncRNAs and their respective neighboring PC genes. Since the location of lncRNAs in the unanchored pseudo-molecule of the $F$. vesca genome is ambiguous, those IncRNAs were not included in the analysis. 2,870 out of 3,099 lncRNA loci have PC neighbors either upstream or downstream within a $10 \mathrm{~kb}$ distance. 1,461 gene pairs showed absolute value of correlation coefficient $>0.5$ (Additional file 12) and are 


\section{a \\ b}
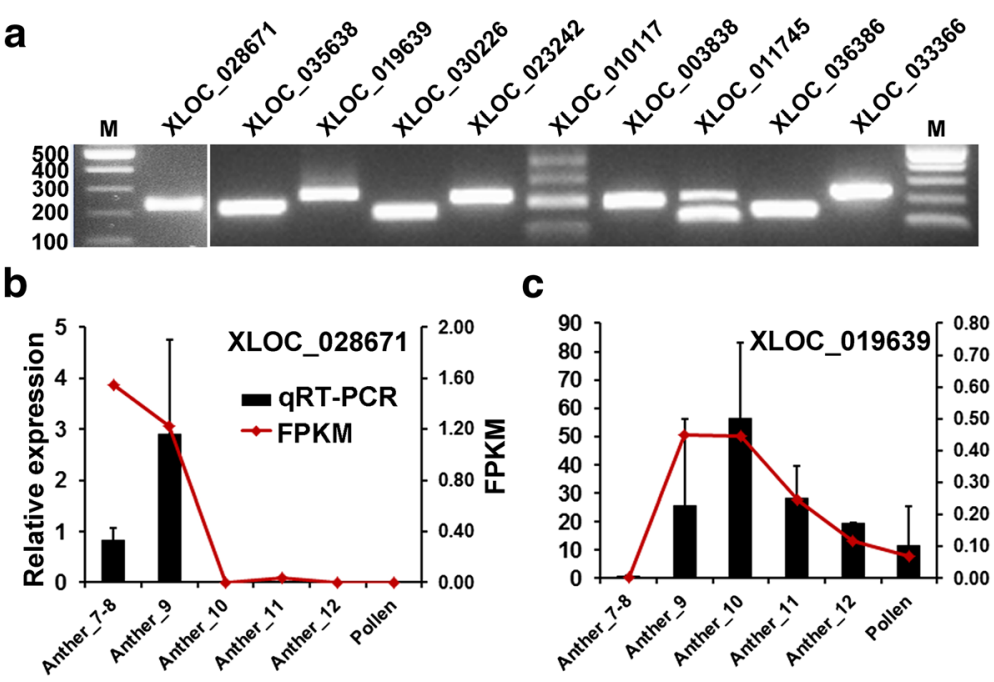

d

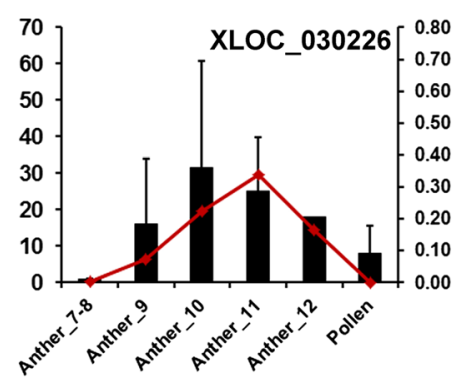

f

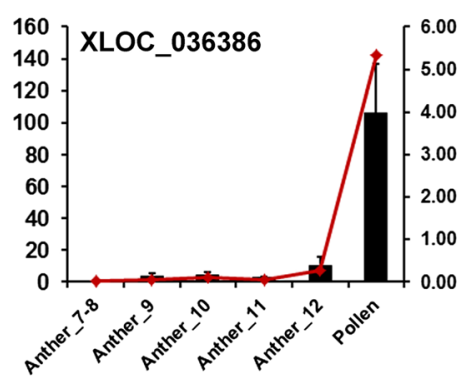

C

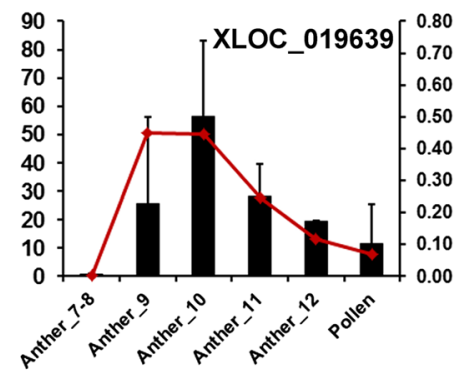

e

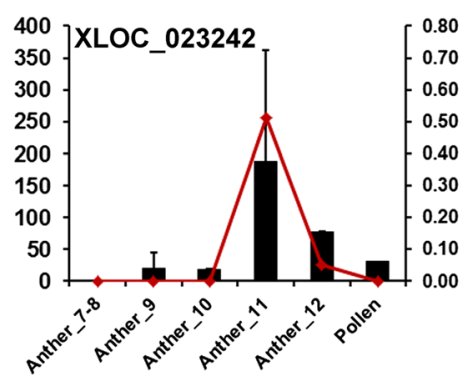

g

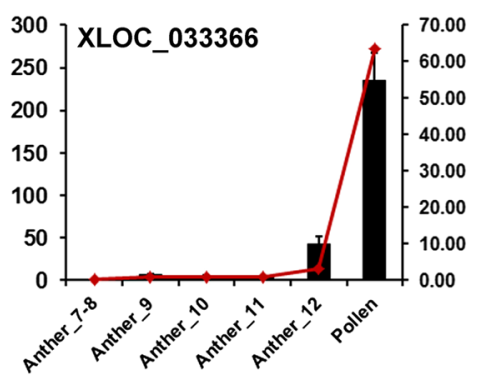

Fig. 3 Validation of anther/pollen specific expression of IncRNAs by qRT-PCR. a Gel image of RT-PCR products of ten randomly selected anther/ pollen specific IncRNAs. $\mathbf{b}$ to $\mathbf{g}$ The expression of six IncRNAs quantified by qRT-PCR (black bar and Y-axis on the left). Error bar indicates standard deviation (SD) of two biological replicates with three technical replicates each. The relative FPKM of the same six IncRNAs based on RNA-seq data was also shown (red line and Y-axis on the right). Gene11892 was used as the internal control for both RNA-seq (red line) and qRT-PCR (black bar). RNAs were from anthers at stage7/8, stage9, stage10, stage11, and stage12 as well as mature pollen

thus promising candidates for cis-regulation. Although lncRNAs were slightly more positively correlated with neighboring coding genes than PC genes with their neighbors in statistics (Kolomogorv-Smirnov (KS) test, $P<0.05$; Additional file 1: Figure S4), the distributions of correlation coefficients for IncRNA-to-neighbor and PCto-neighbor are quite similar, in agreement with findings in human [28]. Further, many more lncRNA-to-neighbor gene pairs showed a positive correlation than those showing a negative correlation (1,422 pairs with $r>0.5$ versus 39 pairs with $\mathrm{r}<-0.5)$. However, an in depth examination of negatively correlated lncRNA-neighbor pairs revealed some intriguing findings. As shown in Fig. 4, XLOC_014500, an intergenic lncRNA locus with three isoforms and two exons, has a correlation coefficient of -0.645 with the neighboring gene XLOC_014501 (gene22438), which codes for a pentatricopeptide repeat-containing protein. The opposite expression pattern is evident in all 37 tissues (Fig. 4a) and is illustrated in detail for the fruit cortex tissues (Fig. 4b). 

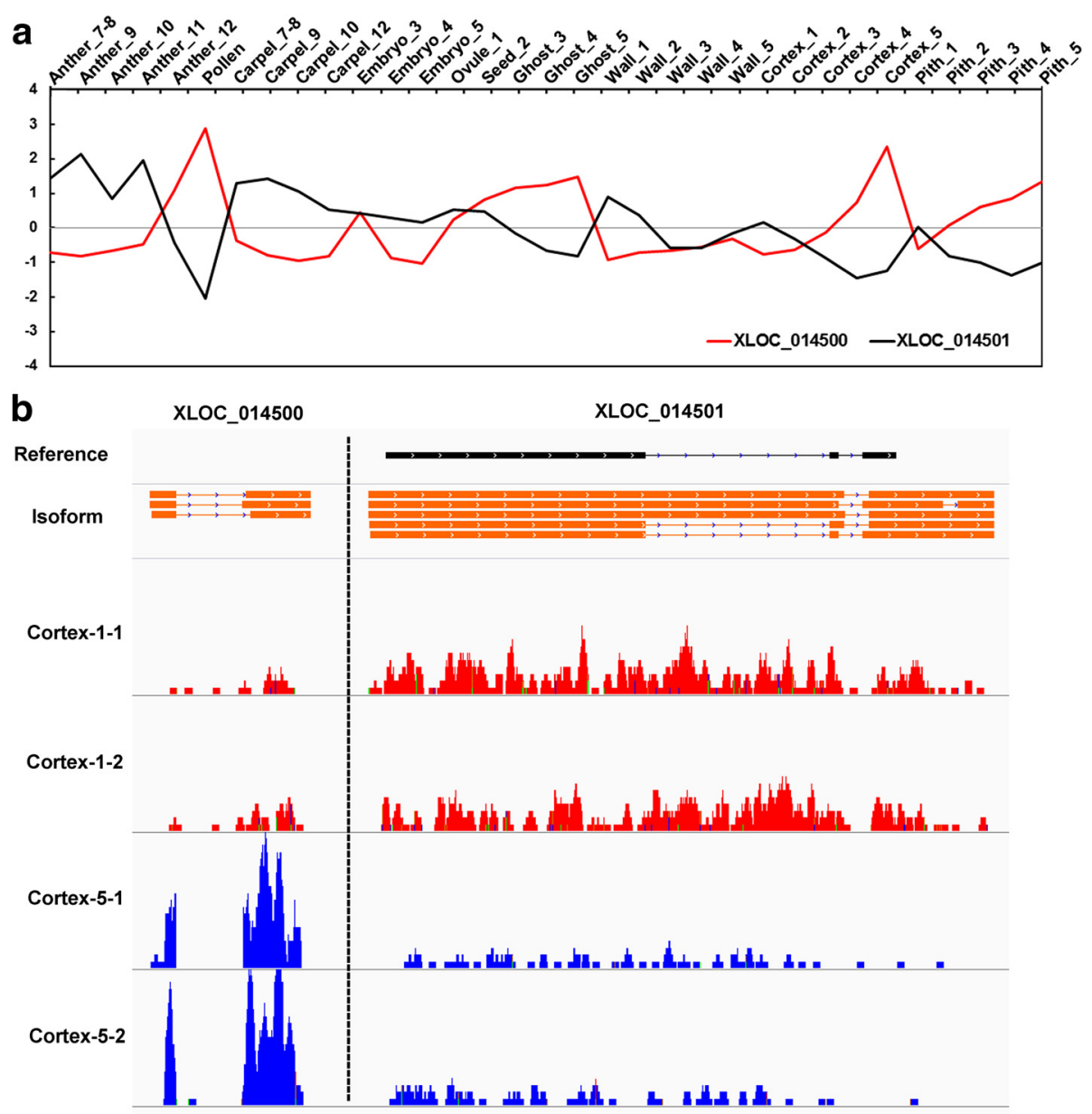

Fig. 4 An example of negative correlation in expression between an IncRNA and its neighboring PC gene. a The expression of IncRNA XLOC_014500 (red line) and neighboring coding gene XLOC_014501 (black line, gene22438) is negatively correlated. Y-axis shows the expression level by Z-score obtained from averaged FPKM of two replicates. $\mathbf{b}$ IGV view of aligned RNA-seq read counts for XLOC_014500 and XLOC_014501 based on two fruit tissue stages: Cortex-1 (pre-fertilization) and Cortex-5 (post-fertilization). The panel of "Reference" shows the gene structure based on genome annotation version1.1. Thin line indicates intron and thick line denotes exon. The panel of "Isoform" shows transcript variants predicted by Cufflinks. The bottom four panels illustrate the RNA-seq read counts in respective tissues. The two replicates are shown with identical color

The lncRNA gene XLOC_014500 is highly expressed in the stage 5 cortex, while XLOC_014501 is lowly expressed in the stage 5 cortex. In contrast, XLOC_014500 is lowly expressed in the stage 1 cortex, while XLOC_014501 is highly expressed in the stage 1 cortex. While the observed expression correlations between fve-lncRNAs and their co-expressed neighbors are highly intriguing, whether they reflect true regulatory relationships or not require further testing.

\section{Expression correlation of IncRNAs with PC genes in trans} Prior reports in animal systems also suggest trans-acting modes of some lncRNAs [12, 50]. To identify lncRNAs that may act in trans, the expression correlations between lncRNAs and all PC genes in the $F$. vesca genome were calculated. 1,330 out of 3,099 lncRNAs were negatively correlated with PC genes ( $r<-0.7$, Additional file 13). 313 lncRNAs each showed negative coefficients with more than 10 genes; the expression patterns of these 313 IncRNAs are shown in the heatmap (Fig. 5a). While a majority of these lncRNAs showed high levels of expression in the pollen, two clusters of lncRNAs ( $\mathrm{C} 1$ and $\mathrm{C} 2$ ) showed complementary expression patterns. One cluster (C2 in Fig. 5a) was specifically and more abundantly expressed in the receptacle fruit (cortex and pith) at post fertilization stages (stages 2-5). The complementary cluster ( $\mathrm{C} 1$ in Fig. 5a) was expressed in other tissues excluding the receptacle (cortex and pith) at stages 2-5. Successful fertilization of ovules has been shown to induce receptacle fruit initiation $[40,51]$, therefore the C2 cluster of lncRNA genes may be induced by fertilization with potential roles in promoting fleshy 


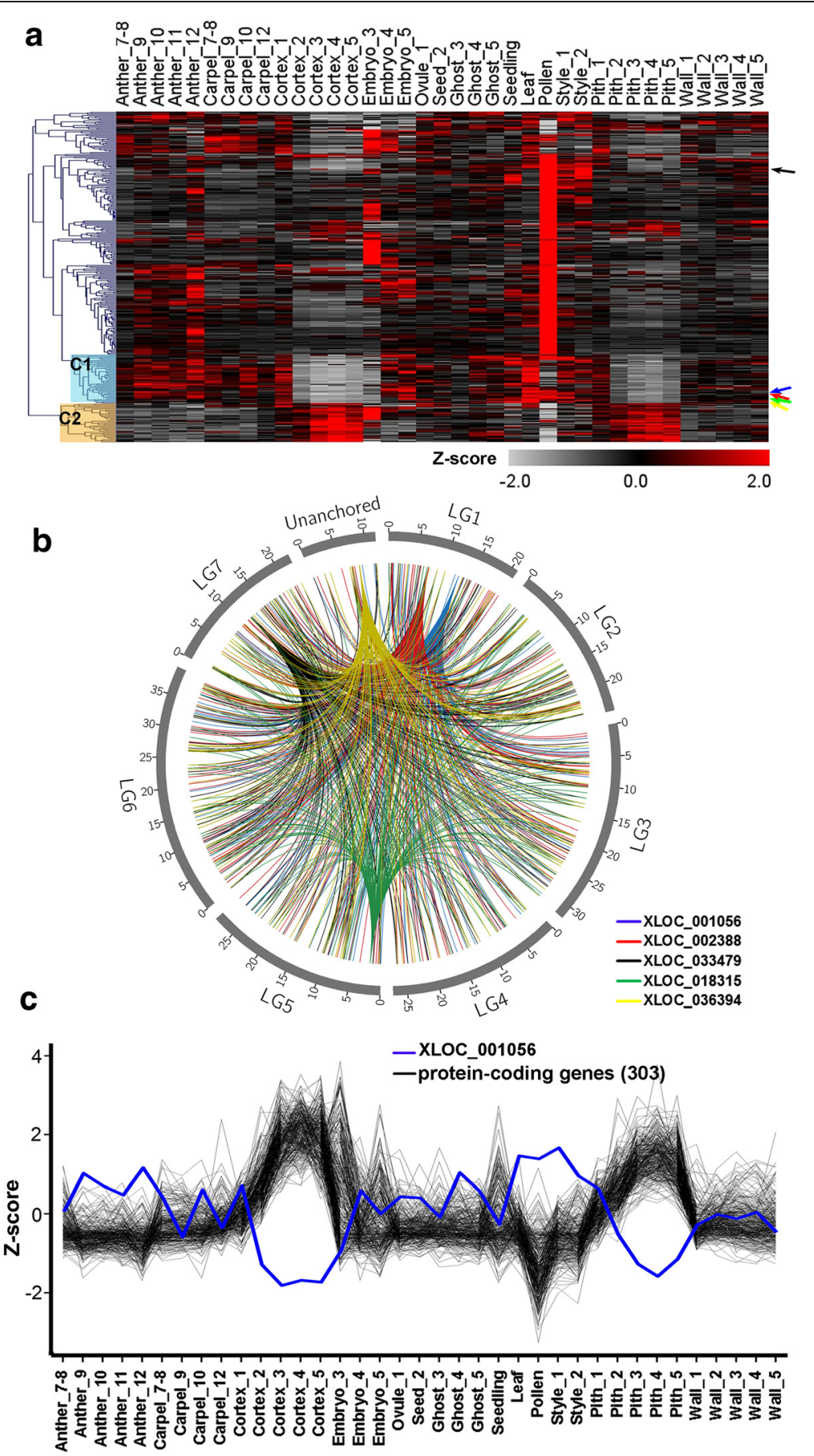

Fig. 5 Negative correlation in expression between IncRNAs and PC genes across the genome. a Heatmap showing the expression of 313 IncRNAs, which were identified as having 10 or more negatively correlated PC genes across the genome at a cut-off of $r<-0.7$. Clusters C1 and C2 were highlighted and are specifically repressed or activated in the receptacle. $\mathbf{b}$ Top five IncRNAs with the highest number of negatively correlated protein coding genes are shown. Each of the five IncRNAs is connected to its negatively correlated protein-coding genes with lines. The expression pattern of each of the five IncRNAs is marked in (a) by arrows with the same colors. $\mathbf{c}$ The opposite expression trend between InCRNA XLOC_001056 (blue line) and its 303 negatively correlated PC genes (black lines). Z-score used in (a) and (c) was obtained from averaged FPKM from two replicates

fruit initiation. In contrast, the C1 cluster of lncRNAs may possess opposite roles and may be involved in repressing receptacle fruit development.
The five lncRNAs with the highest number of negatively co-regulated genes are shown in a graph (Fig. 5b). Each of the five IncRNAs is connected to the co- 
regulated genes in the genome with colored lines. These 5 lncRNAs are all expressed at a low level in the receptacle fruit, with four belonging to the $\mathrm{C} 1$ cluster (Fig. 5a, Additional file 1: Figure S5A). In contrast, their negatively correlated 553 PC genes are more highly expressed in the receptacle (Additional file 13). Fig. 5c illustrates the expression of IncRNA XLOC_001056 and its corresponding 303 negatively co-regulated PC genes. Given that these 5 lncRNAs could potentially contribute to the regulation of such a large number of negatively correlated PC genes, their impact on the development of fruit could be significant. Enriched GO terms for these 553 PC genes include metabolic processes and intracellular transport processes, consistent with functions in fruit tissue development (Additional file 1: Figure S5B, Additional file 13). These analyses not only identified fve-lncRNAs with potential regulatory roles in trans, but also highlighted specific lncRNAs that could potentially serve as hubs in a coordinated gene expression networks underlying receptacle fruit development.

\section{Examination of evolutionary conservation of IncRNAs}

If lncRNAs perform evolutionarily conserved functions, they could be conserved across species even though a lack of coding constraints may enable rapid changes in DNA sequences. Thus, we investigated if any of the fveIncRNAs identified in this study are conserved across different plant species. First, we used the fve-lncRNAs to blast against the genomes of Arabidopsis thaliana, maize (Zea mays), rice (Oryza sativa), apple (Malus domestica), and peach (Prunus persica); 36, 50, 52, 450 and $511 F v e$-lncRNAs were found to share similarities with certain sequences in the respective plant genomes (Evalue $<0.001)$. Since apple, peach, and strawberry belong to the Rosaceae family, they are more closely related to one another. The numbers above suggest that about $1 \%$ of the fve-lncRNAs have potential conserved counterparts in the non-Roseceae species and about $10 \%$ fvelncRNAs have potential homologs in the Roseceae species. Hence, the evolutionary conservation of lncRNA is rather limited.

The above analysis could not determine if the homologous sequence in the other species encode lncRNAs. Therefore, we investigated if the fve-lncRNAs are homologous to lncRNAs already identified in these species. Currently, 6,480 lncRNAs were identified in Arabidopsis [31], 2,224 lncNRAs were identified in rice [35], and several thousand lncRNAs were reported from two studies in Zea mays. Boerner and McGinnis first identified 2,492 maize IncRNAs [30]; Li reported 1704 high confidence lncRNAs and 18,459 pre-lncRNAs in maize [47]. 5,884 fve-lncRNAs were blasted against the lncRNAs from Arabidopsis, rice and maize, only one fve-IncRNA (TCONS_00042468, class_code " $u$ ") was found to share similarity to the prelncRNA transcript TCONS_00012579 in maize (Table 2).

To test conservation of fve-LncRNAs in the Rosaceae species, Malus and Prunus, we took advantage of available ESTs for these two species. We first identified lncRNAs from each species applying the same filtering criteria as for $F$. vesca. Specifically, we analyzed 25,525 assembled unigenes in the Malus unigenes v.5 downloaded from GDR; these unigenes were built previously based on ESTs from mainly Malus domestica. Among them, 4,301 unigenes were found to possess IncRNAs (Additional file 14). 37 of these $M$. domestica unigenes exhibited sequence similarities to $49 F$. vesca lncRNAs (E-value $<0.001$ ) (Table 2). Similarly, analysis of 10,934 assembled unigenes in the Prunus unigenes v.5 (based on ESTs from apricot, peach, Chinese plum, and cherry) yielded 1,315 unigenes that likely produce lncRNAs (Additional file 15). 7 of these Prunus unigenes showed sequence similarity to $19 \mathrm{~F}$. vesca lncRNAs (E-value < 0.001) (Table 2). The conservation of these lncRNAs

Table 2 Identification of conserved IncRNAs in related species

\begin{tabular}{lllll}
\hline $\begin{array}{l}\text { Number of Fve-IncRNAs with } \\
\text { homologs in other species }\end{array}$ & $\begin{array}{l}\text { Number of Arabidopsis IncRNAs } \\
\text { with homologs in other species }\end{array}$ & $\begin{array}{l}\text { Total IncRNAs in other } \\
\text { species }\end{array}$ & Other Species & $\begin{array}{l}\text { Reference for IncRNA-identification } \\
\text { in other species }\end{array}$ \\
\hline $0 / 0$ & $-/-$ & 6,480 & Arabidopsis & Liu et al. [31] \\
$0 / 0$ & $0 / 0$ & 2,492 & Maize & Boerner and McGinnis [30] \\
$0 / 0$ & $0 / 0$ & 1,704 & Maize (hc-IncRNAs) & Li et al. [32] \\
$1 / 1$ & $5 / 27$ & 18,459 & Maize (pre-IncRNAs) & Zhang et al. [35] \\
$0 / 0$ & $5 / 9$ & 2,224 & Rice & This wrok \\
$-/-$ & $0 / 0$ & 5,884 & Fragaria vesca & This work \\
$49 / 37$ & $2 / 2$ & 4,301 & Malus-unigene & This work \\
$19 / 7$ & $0 / 0$ & 1,315 & Prunus-unigene & \\
\hline
\end{tabular}

Notes:

Column 1 shows the number of Fve-IncRNAs with homologs in other (non-Fragaria) species listed in column 4. The number before ' $/$ ' refers to IncRNA number in Fragaria and the number after ' $\%$ ' refers to the number of IncRNA in the target (non-Fragaria) species. The numbers in column 1 were derived from blast searches using strawberry IncRNAs as queries against the target species. The blast E-value cutoff is $<0.001$. The second column shows results using Arabidopsis IncRNAs as queries against corresponding target species. No blast was performed against the query itself and hence was marked as '-/-' 
within the same plant family suggests that these lncRNAs may arise in the common ancestor of these species and may confer biological functions unique to this family of plants.

To investigate possible conservation of lncRNAs across any plant species described above, the 6,480 lncRNAs from Arabidopsis [31] were blasted against the lncRNAs from maize, rice, Malus and Prunus, respectively. Five of these Arabidopsis lncRNAs showed similarity to 27 of 18,459 pre-lncRNAs from maize (E-value $<0.001$ ) [47]. Five Arabidopsis lncRNAs are similar to 9 of the 2,224 IncRNAs from rice (E-value < 0.001) [35]. Two Arabidopsis $\operatorname{lncRNAs}$ are similar to 2 of the 4,301 lncRNAs from Malus (E-value $<0.001$ ). No homolog was identified in other blasts. Therefore, lncRNAs were not well conserved in higher plants, perhaps reflecting the fast evolving nature of lncRNAs due to the lack of constraint normally imposed upon PC genes [5, 52, 53].

\section{Discussion}

LncRNAs are being increasingly recognized as an important class of regulatory molecules in both animals and plants. While lncRNAs have been widely studied in human and animals, they are still poorly studied in plants with the exception of a limited number of model plant species. To date, no lncRNA has been described in strawberry nor in any other Rosaceae species. In this study, we performed genome-wide identification of IncRNAs from diploid strawberry Fragaria vesca as well as several other Rosaceae species including Malus and Prunus, thereby providing a first look at the landscape of noncoding genes in the Rosaceae genomes. Since $F$. vesca possesses a full complement of molecular genetic tools, the discovery of fve-lncRNAs laid the groundwork for future functional studies of lncRNAs.

Since Rosaceae is an important family for fruit crops, the regulation of their flower and fruit development is of considerable interests. Previously we developed extensive floral and fruit tissue transcriptomes for $F$. vesca via RNA-seq and small RNA-seq [41, 42, 48]. In this study, we mined these transcriptome datasets for lncRNAs using a set of stringent filtering criteria. We identified $5,884 F$. vesca lncRNAs coded by 3,862 loci. We showed that these $F$. vesca noncoding loci possess features similar to PC loci; they contain promoters, exons, and introns, and are alternatively spliced. On the other hand, we showed that the IncRNAs are not well conserved when compared with PC genes, have fewer exons, are expressed at lower levels, and are shorter in transcript length (Additional file 1: Figure S2). These findings are consistent with other studies on plant lncRNAs [29-35]. One most striking feature of these lncRNAs is their tissue-specific expression indicating possible function in specific flower or fruit tissues or at specific stages of development.

The lncRNAs identified from our study may represent only a fraction of lncRNAs coded by the $F$. vesca genome. This is because these lncRNAs were only identified from libraries derived from flower and fruit tissues grown under normal conditions. Further, since the RNA-seq libraries were made from polyA-selected mRNAs, nonpolyadenylated lncRNAs were missed from these libraries. Finally, natural antisense transcripts (NATs) transcribed from the presumptive non-coding DNA strand could be missed due to a lack of strandspecific information in our libraries. Therefore, our work only represents the initial genome scale identification of lncRNAs. Nevertheless, the large number of lncRNAs identified in this study suggests that lncRNAs may contribute significantly to strawberry flower and fruit development.

\section{Plant IncRNAs are not well conserved during evolution}

Blast searches of fve-lncRNAs against the lncRNAs identified in several non Rosaceae plant species yielded none or very few homologs (Table 2), indicating limited evolutionary conservation. Though non-coding RNAs evolve faster than PC genes [52], thousands of conserved lncRNAs have been found among primates and several tetrapod species $[5,53]$, possibly owing to more ancient origin of these lncRNAs found in animals and more time to be stabilized in function. In contrast, flowering plants only arose 130 million years ago and their lncRNAs may be of recent origin and maybe relatively young and transient.

It is not surprising why lncRNAs are not well conserved. First, lncRNAs are not constrained by codon usage. Second, lncRNAs may possess short conserved motifs, but these short motifs are not easily identifiable by BLAST. For example, an intergenic lncRNA family Mt4/IPS1/At4/TPSI1/OsPI1 found in multiple plant species [21-24] contains a $\sim 23$ bp short and conserved motif; this motif binds miR-399 via sequence complementarity, the basis of "target mimicry" [25]. Target mimicry is emerging as a prevalent mode of action by lncRNAs since more lncRNAs are found to act through target mimicry [35, 54]. Third, $36 \%$ lncRNAs are probably associated with small RNAs in this study, most of which should be siRNAs (Fig. 1, Additional file 8). The siRNAs could be generated from a short pairing fragment of two RNAs, such as lnc-NATs, or the stem-loop structure of a single RNA, which are less constrained in other parts of the transcripts $[55,56]$. Fourth, some lncRNAs may directly interact with RNA-binding proteins through conserved secondary structures $[9,57]$. 
The expression of IncRNAs is highly tissue-specific

A major challenge in deducing lncRNA function resides in that lncRNAs do not encode proteins. Gene expression, specifically tissue-specific expression, may help shed light on the potential function of these lncRNAs. Pairwise comparisons between tissues or stages revealed that $27.5 \%$ of lncRNA isoforms and $52.3 \%$ of lncRNA loci are differentially expressed and show high tissue specificity (Fig. 2), suggesting that lncRNAs are subject to active transcriptional regulation. Most notably, a large number of lncRNAs are highly and specifically expressed in the mature pollen (Fig. 2). However, PC genes are also highly and specifically expressed in the mature pollen [42]. This similarity may simply reflect that mature pollen is a very unique tissue. Nevertheless, the action of pollen-specific lncRNAs and their interaction with pollen-specific PC genes may underlie the unique characteristics of mature pollen. The precise regulation of lncRNAs in specific tissues support that IncRNAs may play important functions during reproductive development.

\section{Expression correlations of IncRNAs with PC genes in cis and in trans}

In animals and plants, lncRNAs have been shown to act either in cis or in trans to regulate PC gene expression $[7,18,20,49,50]$. Based on the idea that lncRNAs and their regulatory targets may exhibit highly correlative expression either positively or negatively, we sought to identify potential regulatory targets of the fve-lncRNAs by taking advantages of the available RNA-seq data for a large number (thirty-seven) of $F$. vesca tissues. A similar strategy was successfully employed in finding candidate regulatory targets of lncRNAs in mammals and model plants $[5,8]$. Through this strategy, we identified 1,423 positively correlated and 39 negatively correlated pairs of lncRNAs and neighboring genes (Additional file 12, Fig. 5). The positive expression correlations may simply result from their subjecting to common regulations of local chromatin. However, some correlations could reflect authentic regulatory relationships between an lncRNA and its neighboring gene. An encouraging example is the positive expression correlation between lncRNA $A P O L O$ and its regulatory target $P I D$, which codes for a key regulator of polar auxin transport [8]. In our study, we showed an example of an lncRNA XLOC_014500 and its negatively correlated PC gene XLOC_014501 (Fig. 4). XLOC_014501 encodes a protein belonging to the PPR protein family that has RNA binding capacity [58]. The opposite expression pattern and the immediate upstream location of the IncRNA XLOC_014500 with respect to the PPR gene warrants further investigation into a possible direct regulatory relationship between the lncRNA and its neighboring PPR gene.
To identify lncRNAs acting in trans, we performed a correlation analysis between 3,112 lncRNAs and all PC genes in the $F$. vesca genome (Additional file 13). One third of the lncRNAs have negatively correlated PC genes at a cut-off of -0.7; this is not surprising given the pervasive in trans regulations reported in animals [12, 50]. In examining the expression profiles of these lncRNAs, we identified clusters of lncRNAs exhibiting receptacle fruitspecific repression or activation (Fig. $5 \mathrm{a}, \mathrm{C} 1$ and $\mathrm{C} 2$ ). It will be interesting to determine if $\mathrm{C} 1$ and $\mathrm{C} 2$ clusters play opposite roles in the development of this unique strawberry fruit type. Among the lncRNAs that show receptacle-specific repression, we selected 5 that have expression patterns correlated with the highest number of PC genes (Fig. 5b; Additional file 1: Figure S5). The 553 coding genes targeted by these five lncRNAs are enriched in various metabolic processes associated with active cell proliferation and growth (Additional file 1: Figure S5). These results suggest that the receptacle-repressive lncRNAs could serve as the hubs of a gene regulatory network, the repression of which may lead to positive receptacle fruit growth.

\section{Conclusions}

5,884 Fve-lncRNAs derived from 3,862 loci were identified from diploid strawberry Fragaria vesca using the flower and fruit RNA-seq datasets, thereby providing a first look at the landscape of noncoding genes in one fruit crop of the Rosaceae family. The tissue-specific lncRNA expression patterns and the gene expression correlation analysis between lncRNAs and PC genes identified a set of lncRNAs with potential roles in flower and fruit development. The discovery of fve-lncRNAs laid the groundwork for future functional studies of lncRNAs in strawberry.

\section{Methods \\ Description of RNA-seq datasets}

Two transcriptome datasets generated from diploid strawberry YW5AF7 [59] during flower and fruit development were used for IncRNA identification here. One dataset includes two biological replicates of 25 samples representing 5 different fruit tissues at 5 developmental stages (Additional file 2) [41]. The second dataset includes two biological replicates each of 12 samples representing developing anthers and carpels of flowers (Additional file 2) [42]. RNA-seq libraries were made from polyA-selected RNA and sequenced using Illumina HiSeq2000. About 20-40 million single-end, 51 bp reads were obtained per sample (Additional file 2). Both datasets were deposited at Sequence Read Archive (SRA) at NCBI (http://www.ncbi.nlm.nih.gov/sra). The accession numbers are SRA065786 and SRP035308, respectively. 


\section{Identification of novel transcripts}

Sequence reads of each library were aligned individually to the version $1.1 \mathrm{~F}$. vesca genome which has 32,831 annotated PC genes (Fvesca_226.fa and Fvesca_226_gene.gff3 downloaded from http://phytozome.jgi.doe.gov/pz/ portal.html) using the TopHat 2.0 program [60]. During the alignment, the minimal anchor length was set as 5 $(-\mathrm{a})$, the maximal intron length was set as $5000(-\mathrm{I})$, and other settings were at default. Only uniquely aligned reads were used for further analysis ("NH:i:1"). To derive all novel transcripts, the unique reads were assembled individually using the Cufflinks 2.0 program. Next, transcripts with coverage higher than 2 in each library were combined using Cuffmerge and then compared to Fvesca_226_gene.gff3 to assign the class_code to each transcript using Cuffcompare [61]. Finally, Cuffdiff was used to call all the differentially expressed genes in pairwise comparisons ( $q$-value $<0.01$, fold change $>2$ ). The gene expression level at FPKM (Fragments Per Kilobase of exon per Million fragments mapped) was obtained by the CummeRbund $\mathrm{R}$ package, and tissue specificity score (JS score) was calculated for each transcript using the csSpecificity() function in this package.

\section{Filtering strategy used to identify IncRNAs}

Among the assembled transcripts, the majority are partially $(72,727$, class_code "j") or completely $(26,093$, class_code "=") matched with the existing annotation. As the version 1.1 annotation includes only PC genes, these two categories $(j$ and $=$ ) should represent PC genes and were thus excluded from further analysis. The transcripts with class_code "u" (unknown intergenic transcript), "o" (generic exonic overlap with a reference transcript), " $x$ " (natural antisense transcript, NAT), and "i" (intronic transcript) were subjected to PC potential calculation [45]. Non-coding transcripts (coding potential score $(\mathrm{CPC})<-1)$ larger than 200 bp were extracted for further analysis. Transcripts with unknown direction were kept only if both orientations possess no coding potential. Further, transcripts that encode any conserved protein domains were removed in the sense strand for multi-exonic transcripts or in either strand for singleexon transcripts. These transcripts were identified by searching against the Pfam database $(E-v a l u e<0.001)$ [62]. The remaining transcripts were blasted against the Rfam database (http://rfam.xfam.org/), tRNA database (http://gtrnadb.ucsc.edu/), and rRNA database (http:// ssu-rrna.org/) to remove any known transcripts (E-value $<0.001)$. To eliminate all possible pre-miRNAs, transcripts that perfectly match the 362 miRNAs found in the octoploid and diploid strawberries were filtered out [63-65]. To discover lncRNAs from ESTs (Expressed Sequence Tag), the fifth version of Fragaria unigene downloaded from GDR (www.rosaceae.org) was used for filtering following the same pipeline described above. In addition, they were mapped to the $F$. vesca genome by BLAT with at least $95 \%$ sequence identity in the matched region (-minIdentity) and $50 \%$ matched in length.

\section{Conservation of IncRNAs}

To determine conservation of lncRNAs, fve-lncRNAs were blasted against a few plant genomes and lncRNAs from other species using standalone blastn program (blast-2.2.28+, E-value $<0.001$ ). The genomes of Arabidopsis (Arabidopsis_thaliana.TAIR10), maize (Zea_mays.AGPv3), and rice (Oryza_sativa.IRGSP-1.0) were downloaded from the release 28 of the ensemble website (http://plants.ensembl.org/index.html). The genomes of apple (Malus_x_domestica.v3.0.a1) and peach (Prunus_persica_v2.0.a1) were downloaded from GDR. The data resources of lncRNAs used in this study are shown in Table 2 . The fifth version of unigenes from the genera of Malus and Prunus were downloaded from GDR. To discover lncRNAs from Malus and Prunus, their respective unigenes were similarly filtered (length $>200$ bp; CPC $<-1$ ). House-keeping RNAs and conserved miRNAs were removed as well.

\section{Removal of transcripts that can yield small RNAs or contain repetitive sequence}

Raw small RNA-seq reads generated from nine tissue types in woodland strawberry YW5AF7 [48, 59] were previously deposited at the Gene Expression Omnibus (GEO) at NCBI under accession numbers GSE44930 and GSE61798. We re-analyzed the raw reads by quality-filtration (quality score $-q=28$, percent of bases $-p=80 \%$ ), then combined all reads and clipped off the adaptors. The processed reads were collapsed into a single FASTA file by FASTX-Toolkit (http://hannonlab.cshl.edu/fastx_toolkit/). The number of total collapsed reads was $24,405,396$. Only reads of $18 \mathrm{bp}$ to $30 \mathrm{bp}$ in length were used in further analysis. Those reads were aligned to the 5,884 lncRNAs with perfect match by Bowtie1 [66]. The aligned small RNA reads composed of simple repeats or mapped to more than 20 loci were removed. Finally, the lncRNAs were separated into two groups: lncRNAs generating small RNAs $(>10$ reads per alignment) and lncRNAs not generating small RNAs ( $<=10$ reads per alignment).

De novo prediction of repetitive sequences in putative fve-IncRNAs was achieved by RepeatScout with default parameters [67]. Subsequent similarity searches and repeat masking were performed by RepeatMasker (http://www.repeatmasker.org) against both the repeat library obtained by RepeatScout and the Rosaceae repeat library (version 20140131) deposited at the RepBase (http://www.girinst.org/). The lncRNAs were separated into two groups: lncRNAs associated with repetitive sequences $(>=10 \%)$ 
and IncRNAs not associated with repetitive sequences $(<10 \%)$.

\section{Quantitative RT-PCR of IncRNAs}

RNAs were isolated from stage $7 / 8$ to stage 12 anthers and mature pollen using the RNeasy Plant Mini Kit (Qiagen, cat. No.74904) and the RNase-Free DNase Set (Qiagen, cat. No.79254). Two biological replicates were included. cDNA was synthesized from $\sim 1 \mu \mathrm{g}$ total RNA in $20 \mu \mathrm{l}$ solution using iScript $^{\mathrm{Tm}}$ cDNA Synthesis Kit (Bio-Rad Laboratories, Cat. \#170-8891). 5× diluted cDNA was used as the template in real-time PCR analysis. SsoFast ${ }^{\mathrm{Tm}}$ EvaGreen ${ }^{\circ}$ Supermix (Bio-Rad Laboratories, Cat. \#1725203) was used to set up real-time PCR reactions, which were run and analyzed on the CFX96 Real-Time System (Bio-Rad Laboratories). Conditions for real time PCR were: $98{ }^{\circ} \mathrm{C}$ for $3 \mathrm{~min}$, followed by 55 cycles of $95{ }^{\circ} \mathrm{C}$ for $15 \mathrm{~s}$, and $60{ }^{\circ} \mathrm{C}$ for $10 \mathrm{~s}$. Melting curve analysis was performed from $65{ }^{\circ} \mathrm{C}$ to $95{ }^{\circ} \mathrm{C}$ with increments of $0.5{ }^{\circ} \mathrm{C}$. Gene-specific primers are listed in Additional file 13. The Pfaffl formula $2^{-\Delta \Delta C t}$ method was used to calculate relative gene expression differences. Stably expressed gene11892 was used as the internal control [41].

\section{Correlation analysis between IncRNAs and PC genes}

To test the correlation of expression between lncRNAs and their neighboring genes, both upstream and downstream PC genes within $10 \mathrm{~kb}$ distance of the 3,099 lncRNAs were identified by Bedtools (makewindows and intersect) [68]. PC genes that overlap with lncRNAs were not included in this analysis. The Pearson correlation coefficient was calculated by cor() using average FPKM of two replicates in R. To obtain the expression correlation of lncRNAs and distant PC genes, correlation coefficients were similarly calculated between lncRNAs and all the other PC genes excluding known neighbor genes. As too many positive correlations were found, only negative correlations with $\mathrm{r}<-0.7$ were preserved. The $p$-value was calculated by the function corPvalueStudent() in the R package WGCNA [69].

\section{Availability of supporting data}

The data sets supporting the results of this article are included within the article and its additional files. Readers can also visualize $F$. vesca lncRNAs as a track in gBrowse hosted at SGR (http://bioinformatics.towson.edu/strawberry/) as well as at GDR (https://www.rosaceae.org).

\section{Additional files}

Additional file 1: Figure S1. Length distribution of small RNAs derived from IncRNAs. Figure S2. Characterization of strawberry IncRNAs. Figure S3. Heatmaps showing tissue-specific expression patterns of IncRNAs. Figure S4. Expression correlations between IncRNAs and the adjacent PC genes. Figure S5. Negative correlations of IncRNA expression with PC genes across the genome. (PDF $12315 \mathrm{~kb}$ )

Additional file 2: The descriptions and mapping statistics of the $\mathbf{7 4}$ samples. (XLSX $16 \mathrm{~kb}$ )

Additional file 3: The sequences of 5,884 IncRNAs in the .fasta format. (FASTA $5602 \mathrm{~kb}$ )

Additional file 4: The annotation file of 5,884 IncRNAs in the .gtf format (cufflink output). (GTF $3031 \mathrm{~kb}$ )

Additional file 5: Annotations of the 5,884 IncRNAs. (XLSX $336 \mathrm{~kb}$ )

Additional file 6: The sequences of 335 unigenes in the fasta format. (FASTA $223 \mathrm{~kb}$ )

Additional file 7: The annotation file of 335 contigs in the .gff3 format (BLAT output). (GFF3 $19 \mathrm{~kb}$ )

Additional file 8: Categories of the 5,884 IncRNAs classified by expression level, repetitive sequences, and small RNAs. (XLSX $1624 \mathrm{~kb}$ )

Additional file 9: Differentially expressed IncRNA isoforms in pairwise comparisons. (XLSX $3558 \mathrm{~kb}$ )

Additional file 10: Differentially expressed IncRNA loci in pairwise comparisons. (XLSX $2178 \mathrm{~kb})$

Additional file 11: Primers used in qRT-PCR for validation of ten random IncRNAs. (XLSX $8 \mathrm{~kb}$ )

Additional file 12: Expression correlations between IncRNAs and adjacent PC genes. (XLSX $894 \mathrm{~kb}$ )

Additional file 13: Negative correlation between IncRNA expression and all PC genes. (XLSX $2974 \mathrm{~kb}$ )

Additional file 14: LncRNAs in Malus. (FASTA $2792 \mathrm{~kb}$ )

Additional file 15: LncRNAs in Prunus. (FASTA $1011 \mathrm{~kb}$ )

\section{Abbreviations}

LncRNA: Long non-coding RNA; Hc-IncRNA: High-confidence IncRNA; PC: Protein coding; siRNA: Small interfering RNA; Lnc-NAT: Long non-coding natural antisense transcript; GDR: Genome Database for Rosaceae; CPC: Coding potential score; FPKM: Fragments Per Kilobase of exon per Million fragments mapped; DE: Differentially expressed; JS score: JensenShannon specificity score; KS test: Kolomogorv-Smirnov test; EST: Expressed Sequence Tag.

\section{Competing interests}

The authors declare that they have no competing interests.

\section{Authors' contributions}

CK and ZL designed the study; CK carried out the analyses and experiments; CK and ZL participated in writing the manuscript. All authors read and approved the final manuscript.

\section{Acknowledgements}

This work was supported by the National Science Foundation Grant MCB0923913 to Z.L. and by the start-up grant from Huazhong Agricultural University to C.K. (Program No. 2014RC017). We would like to thank Dr. Julie Caruana and Rachel Shahan for comments and edits of the manuscript.

Received: 15 May 2015 Accepted: 6 October 2015

Published online: 19 October 2015

\section{References}

1. Yamada K, Lim J, Dale JM, Chen H, Shinn P, Palm CJ, et al. Empirical analysis of transcriptional activity in the Arabidopsis genome. Science. 2003;302(5646):842-6.

2. Mardis ER. Next-generation DNA sequencing methods. Annu Rev Genomics Hum Genet. 2008;9:387-402.

3. Castel SE, Martienssen RA. RNA interference in the nucleus: roles for small RNAs in transcription, epigenetics and beyond. Nat Rev Genet. 2013;14(2):100-12.

4. Rogers K, Chen X. Biogenesis, turnover, and mode of action of plant microRNAs. Plant Cell. 2013;25(7):2383-99. 
5. Guttman M, Amit I, Garber M, French C, Lin MF, Feldser D, et al. Chromatin signature reveals over a thousand highly conserved large non-coding RNAs in mammals. Nature. 2009;458(7235):223-7.

6. Wierzbicki AT, Haag JR, Pikaard CS. Noncoding transcription by RNA polymerase Pol IVb/Pol V mediates transcriptional silencing of overlapping and adjacent genes. Cell. 2008;135(4):635-48

7. Heo JB, Sung S. Vernalization-mediated epigenetic silencing by a long intronic noncoding RNA. Science. 2011;331(6013):76-9.

8. Ariel F, Jegu T, Latrasse D, Romero-Barrios N, Christ A, Benhamed M, et al. Noncoding transcription by alternative RNA polymerases dynamically regulates an auxin-driven chromatin loop. Mol Cell. 2014:55(3):383-96.

9. Di C, Yuan J, Wu Y, Li J, Lin H, Hu L, et al. Characterization of stressresponsive IncRNAs in Arabidopsis thaliana by integrating expression, epigenetic and structural features. Plant J. 2014;80(5):848-61.

10. Bernard D, Prasanth KV, Tripathi V, Colasse S, Nakamura T, Xuan Z, et al. A long nuclear-retained non-coding RNA regulates synaptogenesis by modulating gene expression. EMBO J. 2010;29(18):3082-93.

11. Cesana M, Cacchiarelli D, Legnini I, Santini T, Sthandier O, Chinappi M, et al. A long noncoding RNA controls muscle differentiation by functioning as a competing endogenous RNA. Cell. 2011;147(2):358-69.

12. Guttman M, Donaghey J, Carey BW, Garber M, Grenier JK, Munson G, et al. lincRNAs act in the circuitry controlling pluripotency and differentiation. Nature. 2011;477(7364):295-300.

13. Sana J, Faltejskova P, Svoboda M, Slaby O. Novel classes of non-coding RNAs and cancer. J Transl Med. 2012;10:103.

14. Klattenhoff CA, Scheuermann JC, Surface LE, Bradley RK, Fields PA, Steinhauser ML, et al. Braveheart, a long noncoding RNA required for cardiovascular lineage commitment. Cell. 2013;152(3):570-83.

15. Herriges MJ, Swarr DT, Morley MP, Rathi KS, Peng T, Stewart KM, et al. Long noncoding RNAs are spatially correlated with transcription factors and regulate lung development. Genes Dev. 2014;28(12):1363-79.

16. Borsani G, Tonlorenzi R, Simmler MC, Dandolo L, Arnaud D, Capra V, et al. Characterization of a murine gene expressed from the inactive $X$ chromosome. Nature. 1991;351(6324):325-9.

17. Brown $\mathrm{CJ}$, Hendrich $\mathrm{BD}$, Rupert JL, Lafrenière RG, Xing $Y$, Lawrence J, et al. The human XIST gene: Analysis of a $17 \mathrm{~kb}$ inactive $X$-specific RNA that contains conserved repeats and is highly localized within the nucleus. Cell. 1992;71(3):527-42.

18. Rinn JL, Kertesz M, Wang JK, Squazzo SL, Xu X, Brugmann SA, et al. Functional demarcation of active and silent chromatin domains in human HOX loci by noncoding RNAs. Cell. 2007;129(7):1311-23.

19. Redon S, Reichenbach P, Lingner J. The non-coding RNA TERRA is a natura ligand and direct inhibitor of human telomerase. Nucleic Acids Res. 2010;38(17):5797-806.

20. Swiezewski S, Liu F, Magusin A, Dean C. Cold-induced silencing by long antisense transcripts of an Arabidopsis Polycomb target. Nature. 2009;462(7274):799-802.

21. Burleigh SH, Harrison MJ. A novel gene whose expression in Medicago truncatula roots is suppressed in response to colonization by vesiculararbuscular mycorrhizal (VAM) fungi and to phosphate nutrition. Plant Mol Biol. 1997;34(2):199-208.

22. Liu C, Muchhal US, Raghothama KG. Differential expression of TPS11, a phosphate starvation-induced gene in tomato. Plant Mol Biol. 1997;33(5):867-74

23. Martín AC, Del Pozo JC, Iglesias J, Rubio V, Solano R, De La Peña A, et al. Influence of cytokinins on the expression of phosphate starvation responsive genes in Arabidopsis. Plant J. 2000;24(5):559-67.

24. Wasaki J, Yonetani R, Shinano T, Kai M, Osaki M. Expression of the OsPI1 gene, cloned from rice roots using CDNA microarray, rapidly responds to phosphorus status. New Phytol. 2003;158(2):239-48.

25. Franco-Zorrilla JM, Valli A, Todesco M, Mateos I, Puga MI, Rubio-Somoza I, et al. Target mimicry provides a new mechanism for regulation of microRNA activity. Nat Genet. 2007;39(8):1033-7.

26. Ding J, Lu Q, Ouyang Y, Mao H, Zhang P, Yao J, et al. A long noncoding RNA regulates photoperiod-sensitive male sterility, an essential component of hybrid rice. Proc Natl Acad Sci U S A. 2012;109(7):2654-9.

27. Guttman M, Garber M, Levin JZ, Donaghey J, Robinson J, Adiconis X, et al. $\mathrm{Ab}$ initio reconstruction of cell type-specific transcriptomes in mouse reveals the conserved multi-exonic structure of lincRNAs. Nat Biotechnol. 2010;28(5):503-10.
28. Cabili MN, Trapnell C, Goff L, Koziol M, Tazon-Vega B, Regev A, et al. Integrative annotation of human large intergenic noncoding RNAs reveals global properties and specific subclasses. Genes Dev. 2011;25(18):1915-27.

29. Xin M, Wang Y, Yao Y, Song N, Hu Z, Qin D, et al. Identification and characterization of wheat long non-protein coding RNAs responsive to powdery mildew infection and heat stress by using microarray analysis and SBS sequencing. BMC Plant Biol. 2011;11:61.

30. Boerner S, McGinnis KM. Computational identification and functional predictions of long noncoding RNA in Zea mays. PLoS One. 2012;7(8):e43047.

31. Liu J, Jung C, Xu J, Wang H, Deng S, Bernad L, et al. Genome-wide analysis uncovers regulation of long intergenic noncoding RNAs in Arabidopsis. Plant Cell. 2012;24(11):4333-45.

32. Li J, Wu B, Xu J, Liu C. Genome-wide identification and characterization of long intergenic non-coding RNAs in ganoderma lucidum. PLoS One. 2014;9(6):e99442.

33. Shuai $P$, Liang D, Tang S, Zhang Z, Ye CY, Su Y, et al. Genome-wide identification and functional prediction of novel and drought-responsive lincRNAs in Populus trichocarpa. J Exp Bot. 2014;65(17):4975-83.

34. Wen J, Parker BJ, Weiller GF. In Silico identification and characterization of mRNA-like noncoding transcripts in Medicago truncatula. In Silico Biol. 2007;7(4-5):485-505.

35. Zhang YC, Liao JY, Li ZY, Yu Y, Zhang JP, Li QF, et al. Genome-wide screening and functional analysis identify a large number of long noncoding RNAs involved in the sexual reproduction of rice. Genome Biol. 2014;15(12):512.

36. Oosumi T, Gruszewski HA, Blischak LA, Baxter AJ, Wadl PA, Shuman JL, et al. High-efficiency transformation of the diploid strawberry (Fragaria vesca) for functional genomics. Planta. 2006;223(6):1219-30.

37. Shulaev V, Sargent DJ, Crowhurst RN, Mockler TC, Folkerts O, Delcher AL, et al. The genome of woodland strawberry (Fragaria vesca). Nat Genet. 2011;43(2):109-16.

38. Tennessen JA, Govindarajulu R, Ashman TL, Liston A. Evolutionary origins and dynamics of octoploid strawberry subgenomes revealed by dense targeted capture linkage maps. Genome Biol Evol. 2014;6(12):3295-313. doi:10.1093/gbe/evu261.

39. Folta KM, Clancy MA, Chamala S, Brunings AM, Dhingra A, Gomide L, et al. A transcript accounting from diverse tissues of a cultivated strawberry. Plant Genome. 2010;3:90-105.

40. Hollender CA, Geretz AC, Slovin JP, Liu Z. Flower and early fruit development in a diploid strawberry, Fragaria vesca. Planta. 2012;235(6):1123-39.

41. Kang C, Darwish O, Geretz A, Shahan R, Alkharouf N, Liu Z. Genome-scale transcriptomic insights into early-stage fruit development in woodland strawberry Fragaria vesca. Plant Cell. 2013;25(6):1960-78.

42. Hollender CA, Kang C, Darwish O, Geretz A, Matthews BF, Slovin J, et al. Floral transcriptomes in woodland strawberry uncover developing receptacle and anther gene networks. Plant Physiol. 2014;165:1062-75.

43. Darwish O, Slovin J, Kang C, Hollender CA, Geretz A, Houston S, et al. SGR an online genomic resource for the woodland strawberry. BMC Plant Biol. 2013;13:223.

44. Jung S, Ficklin SP, Lee T, Cheng CH, Blenda A, Zheng P, et al. The Genome Database for Rosaceae (GDR): year 10 update. Nucleic Acids Res. 2013;42(D1):D1237-44

45. Kong L, Zhang Y, Ye ZQ, Liu XQ, Zhao SQ, Wei L, et al. CPC: assess the protein-coding potential of transcripts using sequence features and support vector machine. Nucleic Acids Res. 2007;35(Web Server issue):W345-349.

46. Huang X, Madan A. CAP3: A DNA sequence assembly program. Genome Res. 1999;9(9):868-77.

47. Li L, Eichten SR, Shimizu R, Petsch K, Yeh CT, Wu W, et al. Genome-wide discovery and characterization of maize long non-coding RNAs. Genome Biol. 2014;15(2):R40.

48. Xia R, Ye S, Liu Z, Meyers B, Liu Z. Novel and recently evolved miRNA clusters regulate expansive F-box gene networks through phasiRNAs in wild diploid strawberry. Plant Physiol. 2015. doi:10.1104/pp. 15.00253.

49. Ørom UA, Derrien T, Beringer M, Gumireddy K, Gardini A, Bussotti G, et al. Long non-coding RNAs with enhancer-like function in human. Cell. 2010;143(1):46-58

50. Huarte M, Guttman M, Feldser D, Garber M, Koziol MJ, Kenzelmann-Broz D, et al. A large intergenic noncoding RNA induced by p53 mediates global gene repression in the p53 response. Cell. 2010;142(3):409-19. 
51. Nitsch JP. Growth and Morphogenesis of the Strawberry as Related to Auxin. Am J Bot. 1950;37(3):211-5.

52. Marques AC, Ponting CP. Catalogues of mammalian long noncoding RNAs: modest conservation and incompleteness. Genome Biol. 2009;10(11):R124.

53. Necsulea A, Soumillon M, Warnefors M, Liechti A, Daish T, Zeller U, et al. The evolution of IncRNA repertoires and expression patterns in tetrapods. Nature. 2014;505(7485):635-40.

54. Wu HJ, Wang ZM, Wang M, Wang XJ. Widespread long noncoding RNAs as endogenous target mimics for microRNAs in plants. Plant Physiol. 2013;161(4):1875-84.

55. Zhang X, Xia J, Lii YE, Barrera-Figueroa BE, Zhou X, Gao S, et al. Genomewide analysis of plant nat-siRNAs reveals insights into their distribution, biogenesis and function. Genome Biol. 2012;13(3):R20.

56. Wang H, Chung PJ, Liu J, Jang IC, Kean MJ, Xu J, et al. Genome-wide identification of long noncoding natural antisense transcripts and their responses to light in Arabidopsis. Genome Res. 2014;24(3):444-53.

57. Wang Y, Fan X, Lin F, He G, Terzaghi W, Zhu D, et al. Arabidopsis noncoding RNA mediates control of photomorphogenesis by red light. Proc Natl Acad Sci U S A. 2014;111(28):10359-64.

58. Barkan A, Small I. Pentatricopeptide repeat proteins in plants. Annu Rev Plant Biol. 2014;65:415-42.

59. Slovin JP, Schmitt K, Folta KM. An inbred line of the diploid strawberry Fragaria vesca f. semperflorens for genomic and molecular genetic studies in the Rosaceae. Plant Methods. 2009:5:15. doi:10.1186/1746-4811-5-15.

60. Kim D, Pertea G, Trapnell C, Pimentel H, Kelley R, Salzberg SL. TopHat2: accurate alignment of transcriptomes in the presence of insertions, deletions and gene fusions. Genome Biol. 2013;14(4):R36.

61. Trapnell C, Williams BA, Pertea G, Mortazavi A, Kwan G, van Baren MJ, et al. Transcript assembly and quantification by RNA-Seq reveals unannotated transcripts and isoform switching during cell differentiation. Nat Biotechnol. 2010;28(5):511-5

62. Finn RD, Bateman A, Clements J, Coggill P, Eberhardt RY, Eddy SR, et al. Pfam: the protein families database. Nucleic Acids Res. 2014;42(Database issue):D222-230

63. Ge A, Shangguan L, Zhang X, Dong Q, Han J, Liu H, et al. Deep sequencing discovery of novel and conserved microRNAs in strawberry (Fragariaxananassa). Physiol Plant. 2013;148(3):387-96.

64. Xu X, Yin L, Ying Q, Song H, Xue D, Lai T, et al. High-throughput sequencing and degradome analysis identify miRNAs and their targets involved in fruit senescence of fragaria ananassa. PLoS One. 2013;8(8):e70959.

65. Li H, Mao W, Liu W, Dai H, Liu Y, Ma Y, et al. Deep sequencing discovery of novel and conserved microRNAs in wild type and a white-flesh mutant strawberry. Planta. 2013;238(4):695-713.

66. Langmead B, Trapnell C, Pop M, Salzberg SL. Ultrafast and memory-efficient alignment of short DNA sequences to the human genome. Genome Biol. 2009;10(3):R25

67. Price $A L$, Jones NC, Pevzner PA. De novo identification of repeat families in large genomes. Bioinformatics. 2005;21 Suppl 1:i351-358.

68. Quinlan AR, Hall IM. BEDTools: a flexible suite of utilities for comparing genomic features. Bioinformatics. 2010;26(6):841-2.

69. Langfelder P, Horvath S. WGCNA: an R package for weighted correlation network analysis. BMC Bioinform. 2008;9:559.

\section{Submit your next manuscript to BioMed Central and take full advantage of:}

- Convenient online submission

- Thorough peer review

- No space constraints or color figure charges

- Immediate publication on acceptance

- Inclusion in PubMed, CAS, Scopus and Google Scholar

- Research which is freely available for redistribution

Submit your manuscript at www.biomedcentral.com/submit 